\title{
A case of left atrial haemangioma: echocardiographic, surgical, and morphological
} features

\author{
IAN WEIR, * PETER MILLS, † TERENCE LEWIS * \\ From the $\dagger$ Department of Cardiology and the ${ }^{\star}$ Department of Cardiothoracic Surgery, the London Hospital, \\ Whitechapel, London
}

SUMMARY A slow growing encapsulated haemangioma of the left atrium was diagnosed by means of cross sectional echocardiography. The heterogenous nature of the tumour was reflected in a central area of relative echolucency. After successful surgical removal of the tumour, the patient's symptoms of lethargy and limited exercise capacity improved considerably.

\section{Case report}

A sixty three year old woman presented with a fourteen year history of fatigue and shortness of breath. When she was first seen a late systolic murmur had been noted and a diagnosis of mitral regurgitation was made. During follow up the signs remained largely unchanged apart from the development of atrial fibrillation which was treated with digoxin. Her symptoms of fatigue and breathlessness gradually became worse, however, and the murmur was noted to have become louder and longer. Because of progressive enlargement of the cardiac silhouette on chest $x$-ray she was admitted to hospital for further investigation.

On admission she reported breathlessness after walking 100 yards on the flat. She had noticed palpitation, especially on change of posture. Physical examination showed that she was in controlled atrial fibrillation with pronounced right and left parasternal impulses. On auscultation there was a loud first sound and a normally split second sound; a late systolic murmur which varied markedly with posture, was loudest at the apex, and radiated to the axilla. There were no stigmata of bacterial endocarditis or signs of heart failure.

Requests for reprints to Mr Ian Weir, FRCS, Department of Cardiothoracic Surgery, The London Hospital, Whitechapel, London E1 1BB.
Full blood count was normal; 3\% reticulocytes were found. The erythrocyte sedimentation rate was $48 \mathrm{~mm} / \mathrm{h}$. On chest $x$ ray the left atrium seemed to be enlarged. The electrocardiogram showed atrial fibrillation and widespread non-specific $T$ wave changes.

An exercise test was terminated at the end of stage 2 of the modified Bruce protocol because of considerable breathlessness and ventricular bigeminy.

At cardiac catheterisation pressures $(\mathrm{mm} \mathrm{Hg})$ were as follows: pulmonary capillary wedge, $\mathrm{V}=25$, mean 12; pulmonary artery, 35/15; right ventricle, $35 / 8$; right atrium, mean 7 ; aorta, $160 / 60$; left ventricle, $160 / 12$. There were no aortic or mitral valve gradients.

It was noted that the right heart catheter was displaced to the right as it traversed the right atrium.

Coronary angiography showed a normal myocardial circulation from the right and left coronary systems but injections from both the right and left showed an abnormal circulation in the region of the atria.

Cross sectional echocardiography showed a mass $4 \times 6 \mathrm{~cm}$ in the region of the interatrial septum. The mass was immobile and multiple echolucent areas suggested a "soft" or cystic centre (fig 1). In view of these findings it was felt that the left atrium should be surgically explored.

At operation we found a spongy lobulated mass $15 \mathrm{~cm} \times 10 \mathrm{~cm}$ arising from the epicardial surface of 


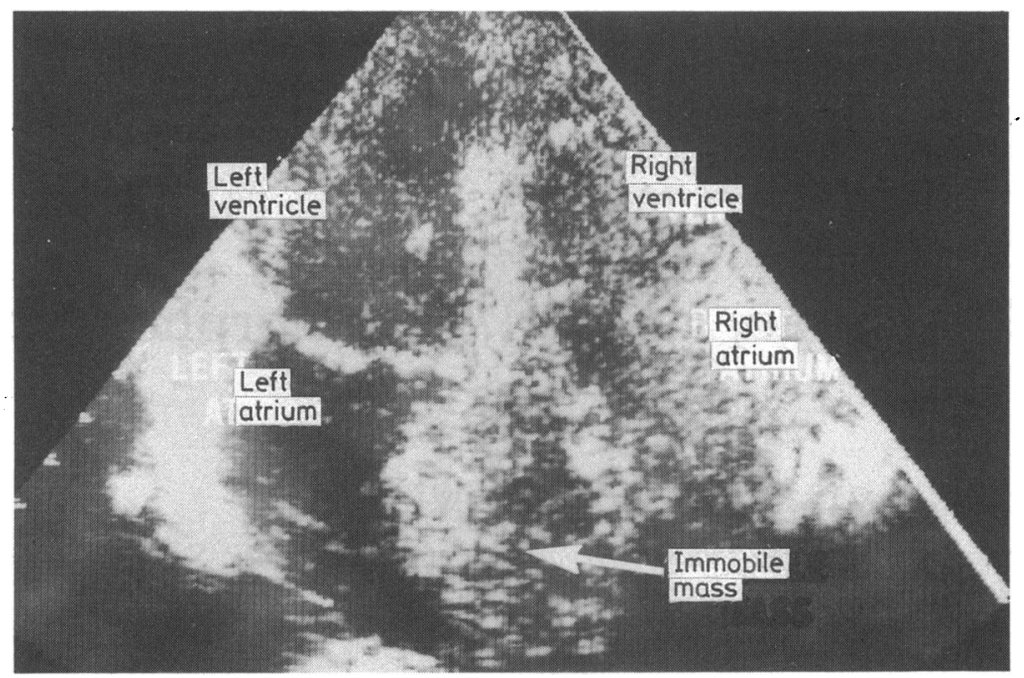

Fig 1 Four chamber cross sectional echocardiogram showing tumour mass in region of interatrial septum.

the posterior wall of the left atrium close to the orifices of the superior pulmonary veins and tracking anteriorly from the transverse sinus medial to the superior vena cava. An additional separate mass $(2 \mathrm{~cm} \times 2 \mathrm{~cm} \times 1 \mathrm{~cm})$ of the same consistency arose from the anterior surface of the aortic root and extended into the atrioventricular groove.

The left atrium was explored on cardiopulmonary bypass. It was found that the tumour had not arisen from within the atrium but rather had been compressing it from without. The mitral valve showed slight prolapse but this was not considered to be sufficiently severe to warrant repair. The visceral pericardium which was encapsulating the tumour was incised and the haemangioma was enucleated with some difficulty. The patient made an uneventful recovery and her symptoms of fatigue and limited exercise capacity improved considerably. She remains well five years after operation.

\section{HISTOLOGY}

Macroscopically the excised mass measured $14 \mathrm{~cm} \times$ $10 \mathrm{~cm} \times 4.5 \mathrm{~cm}$ and was spongy in consistency (fig 2). It was divided into ill-defined lobules by fibrous septa and these lobules were composed of a meshwork of small blood-filled spaces. There was a more centrally placed yellow firmer area measuring $8 \mathrm{~cm}$ $\times 3 \mathrm{~cm}$ situated towards one end of the specimen.

Light microscopy showed a benign haemangioma of the atrium. The predominantly spongy areas of the tumour were composed of large vascular spaces lined by flattened endothelium and in some areas these contained elastic and other collagen fibres in their walls. The more solid yellow area was made up of loose myxoid tissue containing small vessels.

The separate specimen removed from the aortic root had a similar histological appearance as the main tumour mass.

\section{Discussion}

Primary tumours of the heart are rare-according to McAllister and Fenoglio in 1978 there were fewer than 1000 published reports of cases of cysts and neoplasms of the heart and pericardium. ${ }^{1}$ The analysis of 533 of their own cases by these workers showed that $76 \%$ were benign and that the commonest tumour was a myxoma, accounting for $25 \%$ of all such lesions of the heart and pericardium. A haemangioma is the commonest vascular tumour arising from the heart but accounts for only approximately $5 \%$ of all benign cardiac tumours in adults. ${ }^{12}$

In 1975 Tabry et al, reporting a case similar to our own, found only 35 cases of haemangioma of the heart reported throughout the world. ${ }^{3}$ Since these reports, we have found only one further case-a right ventricular haemangioma in a fifteen year old girl. ${ }^{4}$ This last case showed cross sectional echocardiographic features similar to those of our patient but the tumour was found to be inoperable.

Three out of fifteen cases reported by McAllister and Fenoglio presented with symptoms similar to those occurring with myxomas and at necropsy had 


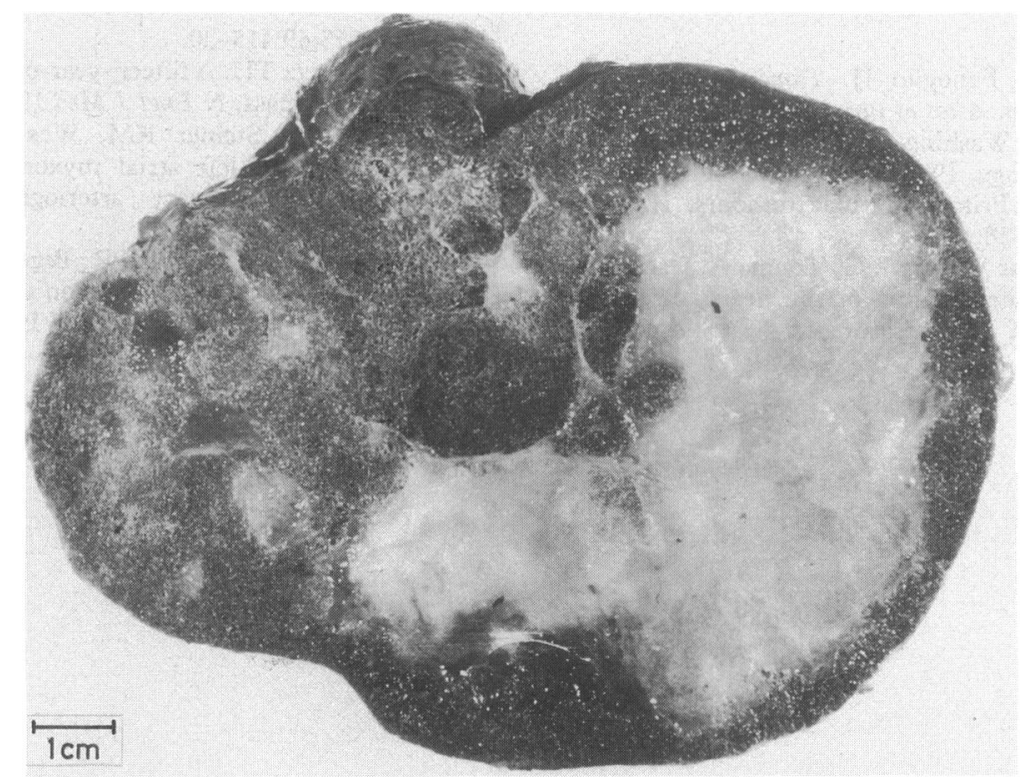

Fig 2 Cut surface of surgically excised tumour showing ill-defined lobules and distinct central myxoid area.

a tumour protruding into a cardiac chamber- the right atrium in two cases and the left ventricle in a third. In two a mass was discovered on routine chest $x$ ray and they were operated on with a suspected diagnosis of pericardial cyst. Histologically, haemangiomas of the heart are identical with haemangiomas occurring at other sites in the body. They may consist of closely packed capillary structures or widely dilated vascular channels lined by flattened endothelial cells and with focal connective tissue in the walls- the pattern of cavernous haemangioma-as seen in the present case.

Cardiac tumours are rare, but the commonest, myxomas, can be diagnosed because their intrinsically mobile nature aids in their detection by echocardiography. By contrast, haemangiomata, which are much less common, are sessile. This explains why in the present case $M$ mode echocardiography could not distinguish the haemangioma from an artefact. Cross sectional study showed a mass $4 \mathrm{~cm} \times$ $6 \mathrm{~cm}$ in the region of the interatrial septum. A striking feature was the lack of homogeneity of the reflected ultrasound from the mass which had an apparently echolucent centre. Morphologically these findings were found to correspond with a large area in which the dense sinusoidal tumour had apparently undergone spontaneous degeneration leaving a loose stroma. Similar structural and echocardiographic features have been previously described ${ }^{4}$ and may represent a means of recognising such tumours on ultrasound.
In our patient, radiological evidence suggested that the tumour had grown slowly and progressively over a period of ten years. It was completely encapsulated, in contrast with the typical friable, gelatinous composition of myxomas. There had been no clinical evidence to suggest embolism seen with unencapsulated tumours in the left atrium.

There was no evidence of major haemodynamic disturbance in our patient. A late systolic murmur was consistently noted over the years. We found that the intensity of this murmur varied strikingly with posture, being much louder when the patient was upright. In addition, a formal exercise test was terminated in stage 2 of the Bruce protocol because of ventricular bigeminy. These features suggest that the tumour may have affected cardiac function in the upright position by impairing left atrial emptying. Postoperatively there was a great subjective improvement in her exercise tolerance and she reached the end of stage 4 of the Bruce protocol.

Intra-atrial masses derive a blood supply from the coronary circulation. Long standing left atrial thrombosis in patients with rheumatic mitral disease may be revealed as a "blush", especially from the left coronary injection. Myxomas too have a vascular supply from the coronary arteries. ${ }^{56}$ The appearance of the coronary angiogram of our patient showed the sinusoids of the haemangioma filling at the same time as the coronary venous phase.

We thank Dr P A Revell for his help. 


\section{References}

1 McAllister HA, Fenoglio JJ. Tumors of the cardiovascular system. Atlas of tumor pathology; Fasicle 15, Second Series. Washington DC: Armed Forces Institute of Pathology, 1978.

2 Silverman NA. Primary cardiac tumours. Ann Surg 1980;191:127-38.

3 Tabry IF, Nassar VH, Rizk G, Touma A, Dagher IK. Cavernous haemangioma of the heart: case report and review of the literature. $J$ Thorac Cardiovasc
Surg 1975;69:415-20.

4 Ryan TJ, Aretz TH. A fifteen-year-old girl with a right ventricular mass. $N$ Engl J Med 1983;308:206-14.

5 Marshall WH, Steiner RM, Wexler L. "Tumour vascularity" in left atrial myxoma, demonstrated by selective coronary arteriography. Radiology 1969;93:815-6.

6 Berman ND, McLaughlin PR, Bigelow WG, Morch JE. Angiographic demonstration of blood supply of right atrial myxoma. Br Heart $J$ 1976;38:764-6. 